\title{
WHY THE TERMS 'CURRENT MODE' AND 'VOLTAGE MODE' NEITHER DIVIDE NOR QUALIFY CIRCUITS
}

\author{
Hanspeter Schmid \\ Bernafon AG, Morgenstrasse 131, 3018 Bern, Switzerland. \\ E-mail: h.p.schmid@ieee.org, WWW: http://www.schmid-werren.ch/hanspeter/
}

\begin{abstract}
It is often stated in papers that there may be a fundamental difference between current-mode and voltage-mode circuits. In this discussion paper, we show that there is no definition that would clearly divide all circuits into current-mode and voltagemode. Then we provide evidence that a voltage-mode $\mathrm{Gm}-\mathrm{C}$ filter and its current-mode counterpart have the same performance. The reason why current-mode circuits often perform differently from voltage-mode circuits is that current-mode circuits often use less loop gain and are less complex. Because one can also build voltage-mode circuits in that way, and current-mode circuits with more gain and complexity, the actual difference between current mode and voltage mode comes from the different preferences of the research groups. We conclude that conscious efforts should be made to re-integrate the knowledge produced by the current-mode research groups into main-stream analog IC design.
\end{abstract}

\section{INTRODUCTION}

Originally, the term "current-mode processing" was coined by Barrie Gilbert when he worked on strict trans-linear loops (c.f. [1]), in which the voltages truly are incidental. Nowadays we are persuaded that current-mode integrators, filters, and oscillators have some special merit. But is this really true? In contrast to strict trans-linear loops, these circuits rely on an intimate dialogue between voltage signals and current signals.

When comparing current-mode to voltage-mode filters, one should perhaps first ask two questions:

First question: Are there any definitions of current mode that make a clear divide between voltage-mode and current-mode circuits? We will call this a precise definition from here on.

Second question: Is it necessary to have a precise definition?

Our answer to both questions is no.

First, none of the definitions used in the literature are precise. For example, some authors write that signals are represented by currents in current-mode circuits and by voltages in voltage-mode circuits. This is not a precise definition, because every circuit node has an associated voltage and every branch an associated current, and it is a matter of definition which ones represent signals and which ones do not. It does not seem possible to refine this definition such that it still includes all kinds of signal-processing circuits.

To give a simple example, it is sometimes said that a current mirror is a current-mode circuit, since the currents have a linear relation to the signal, which the voltage over the diode-connected transistor has not. This argument, however, gives problems as soon as it is applied to log-domain filters. Although one could refine the definition again, and again, ad infinitum, doing this is useless, because the definition will ultimately become a list that states for every imaginable class of circuits whether it operates in the current mode or the voltage mode. Such a list cannot be used to derive general statements about current-mode and voltage-mode circuits.

Nevertheless, the current-mode approach to IC design proposed in [1] and in many newer papers has clearly had a great impact on IC design. Several new circuits and amplifiers (e.g., the operational floating conveyor or the current-feedback OTA [2]) emerged from this way of thinking. We think that the success of the current-mode idea did not occur in spite of the lack of a precise definition, but because of the lack of a precise definition. In other words, reading the papers of the current-mode community does not enable the reader to explicitly define the current-mode approach, but it enables the reader to apply it. This is because, from a certain level of complexity on, an implicit definition can convey much more information than any explicit definition possibly could [3]. An implicit definition can, however, not make it possible to clearly fence off current mode from voltage mode.

Should such a clear divide be made? We think not. The current-mode approach is mainly an alternative way of looking at analog IC design, and not a tool to classify circuits. Taken in this sense, the current-mode idea is a powerful concept mainly because it broadens the horizon of analog IC design. A clear definition would actually destroy its explanatory power.

In the following technical sections of this paper, we will support the views presented in the introduction with two technical discussions. First, we will discuss a recent comparison of a currentmode and a voltage-mode $\mathrm{Gm}-\mathrm{C}$ filter [4]. That discussion showed a slight advantage of the voltage-mode filter, but we will point out an omission made in [4] which, if corrected, shows that the current-mode and the voltage-mode filter have the same performance if they are built with the same technique.

However, there are indeed performance differences between published voltage-mode and current-mode circuits. The reason for this is that voltage-mode and current-mode circuits are often built with different design techniques. Voltage-mode circuits often use higher loop gains than current-mode circuits, and current-mode circuits are often made less complex than the voltage-mode circuits they are compared to. We will discuss this in Section 3, and we will argue that this is not a technical necessity, but simply the designers' preferences.

The conclusion of the whole discussion is: one should not use a current-mode vs. voltage-mode distinction to divide all circuits in two classes. It would, however, be beneficial for voltage-mode designers to study the work of the current-mode community, because there is much to learn from the results provided by their different approach to analog IC design. 


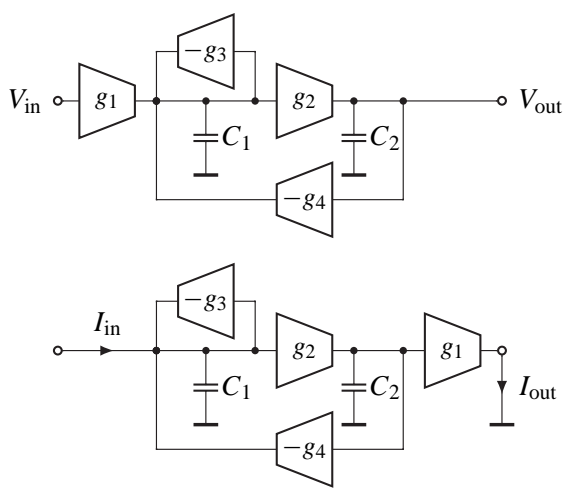

Figure 1: Voltage-mode and current-mode Gm-C filter [4].

\section{OTHER THINGS BEING EQUAL ...}

To find out whether it makes a difference to represent signals by currents instead of voltages, a ceteris paribus (other things being equal) comparison must be made. To our knowledge, only one attempt do do this was published at all [4]. There the authors thoroughly compared the two filters shown in Fig. 1, which are not dual, but have the same loop structure and the same lowpass transfer function. ${ }^{1}$ The study takes into account the noise of the OTAs and the harmonic distortion induced by their non-linear transconductances, but not clipping effects caused by output stage saturation. It is shown that both circuits have similar amounts of harmonic distortion. The circuits are compared with a figure of merit,

$$
F=\frac{\mathrm{DR} \cdot f_{\mathrm{p}}^{2}}{P^{2}}
$$

where DR is the dynamic range, $f_{\mathrm{p}}$ is the pole frequency, and $P$ is the power consumption. The result shows that the dynamic range of the voltage-mode filter is better by at most $6 \mathrm{~dB}$ in the relevant range of pole Qs and DC gains of the low-pass filter function.

The problem with [4] is that it is not really ceteris paribus. The difference mainly occurs because the noise of the input OTA in the voltage-mode filter is processed by the filter, which is not the case for the noise of the output OTA in the current-mode filter. Obviously, if both the input OTA of the voltage-mode filter and the output OTA of the current-mode filter were ideal and noiseless, both filters would perform identically.

The problem with the study in [4] is that the voltage-mode filter has a high-impedance output and the current-mode filter has a high-impedance input. Therefore, on a real IC, the voltagemode circuit needs an output buffer, since a resistive load connected to the output node would otherwise change the transfer function, and the current-mode circuit needs an input buffer, since the input nodes must be driven by a high-resistance device. However, this time the noise of the current buffer is filtered, but not the noise of the voltage buffer, and the performance difference between the two filters is reduced to the performance difference between the circuits used to insert signals into the feedback loop and extract signals from it. The resulting performance difference

\footnotetext{
${ }^{1}$ Actually the filter in Fig. 1 realizes two transfer functions. Choosing the node to which $C_{1}$ is connected as the output node (top) or input node (bottom) results in a bandpass transfer function, but all that is said in this section applies to the bandpass filters as well.
}
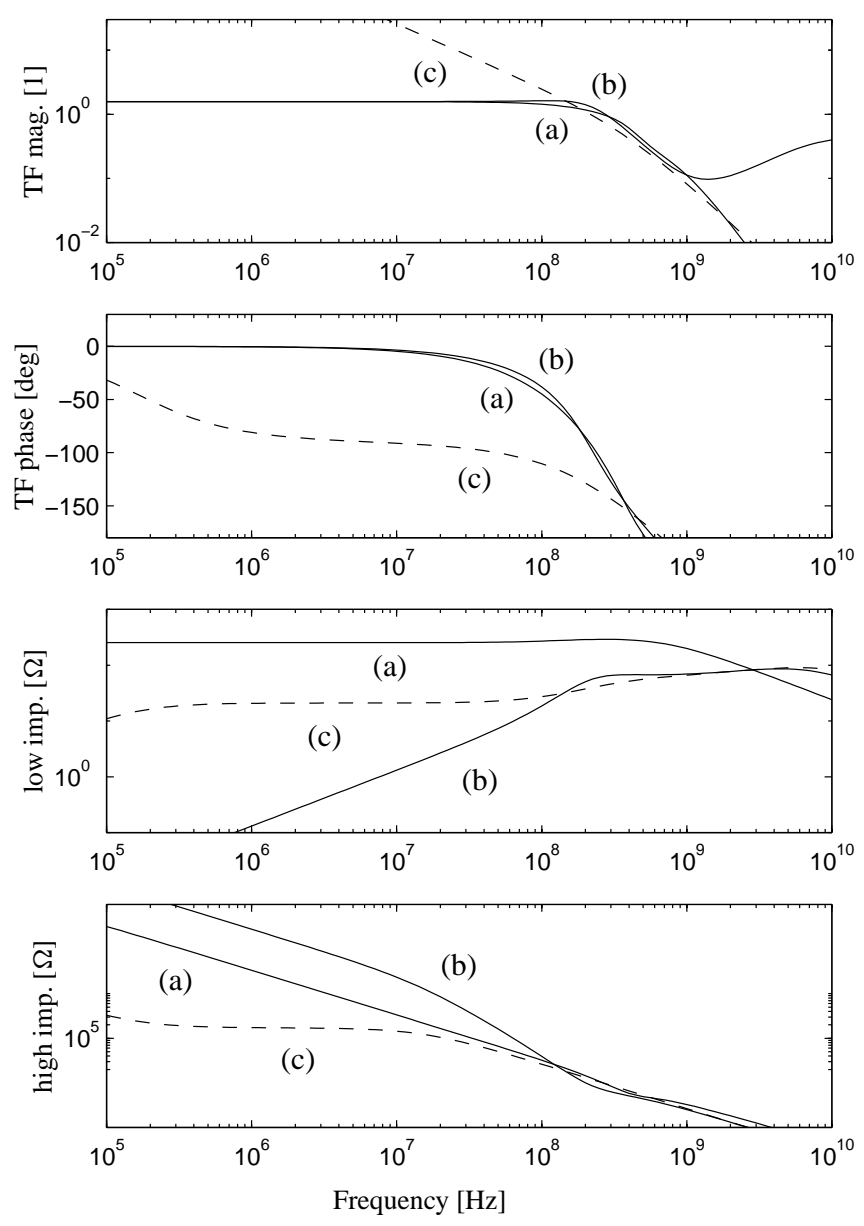

Figure 2: Transfer functions and terminal impedances of (a) the CCII+ in [9], the AD SSM 2135 opamp (b) connected as a voltage buffer and (c) open-loop. The AD SSM 2135 is actually an audio opamp, its frequency has been multiplied by a factor of 50 to make the curves comparable.

is certainly small, and it is not a question of signal representation, but of transistor-level design. Thus, [4] establishes that, other things being equal, there is no performance difference between the current-mode and the voltage-mode Gm-C filter discussed in the paper.

\section{WHICH THINGS ARE NOT EQUAL?}

In most of the papers proposing very fast current-mode circuits, open-loop current amplifiers are compared to results obtained with closed-loop voltage amplifiers [5-8]. Many of the amplifiers derived with a current-mode approach base on current mirrors and provide a specific, low gain without feedback around the amplifier. The typical low-gain voltage amplifier uses feedback around a high-gain amplifier. This feedback stabilises the gain and reduces harmonic distortion, it also improves the terminal impedances of the amplifier.

Fig. 2 shows the transfer functions and terminal impedances of 
a second-generation current conveyor (a CCII+ presented in [9]; c.f. Appendix for a description) and Analog Device's SSM 2135 audio opamp connected as a buffer and in an open-loop configuration. The frequency of the opamp has been multiplied by 50 to make the curves comparable. A look at the transfer functions shows that the closed-loop transfer function of the opamp and the open-loop current transfer function of the CCII+ look very similar. The reason is that both transfer functions are determined by non-dominant poles only, the dominant pole of the voltage opamp, whose effect is clearly visible in the open-loop transfer function, only plays a stabilising role once the feedback loop is closed (see below). The impedance curves show that feedback decreases the output impedance of the opamp but increases the input impedance, which can be considered an improvement in both cases. However, close to the unity-gain frequency $f_{1}$ of the opamp, there is some peaking, which means that feedback actually makes the impedances worse above approximately $f_{1} / 5$. Note that the output impedance of the opamp is far below the input impedance of the CCII+ because the former is built in a bipolar technology, but the latter in CMOS. Although Fig. 2 only shows two specific devices, the effects discussed are the same for other amplifiers.

Coming back to gain stabilisation, the low-gain amplifiers that are used in many filter and regulator circuits are normally built using one voltage opamp and two resistors, as in Fig. 3. Its transfer function is then

$$
T(s)=\frac{V_{\text {out }}}{V_{\text {in }}}=\frac{A(s)}{1+\frac{R_{1}}{R_{1}+R_{2}} A(s)} .
$$

For very high gains,

$$
\lim _{A(s) \rightarrow \infty} T(s)=1+\frac{R_{2}}{R_{1}}=\alpha_{\mathrm{V}},
$$

and if the gain of the amplifier is expressed by the gain-bandwidth product, $A(s) \approx \omega_{\mathrm{gbw}} / s$,

$$
T(s)=\frac{\alpha_{\mathrm{V}} \omega_{\mathrm{gbw}}}{\alpha_{\mathrm{V}} s+\omega_{\mathrm{gbw}}} .
$$

Calculating the relative sensitivity of the transfer function to variations in the gain-bandwidth product gives:

$$
S_{\omega_{\mathrm{gbw}}}^{T(s)}=\frac{d T(s)}{d \omega_{\mathrm{gbw}}} \cdot \frac{\omega_{\mathrm{gbw}}}{T(s)}=\frac{\alpha_{\mathrm{V}} s}{\alpha_{\mathrm{V}} s+\omega_{\mathrm{gbw}}} .
$$

Similarly,

$$
S_{\alpha \mathrm{V}}^{T(s)}=\frac{\omega_{\mathrm{gbw}}}{\alpha_{\mathrm{V}} s+\omega_{\mathrm{gbw}}} .
$$

For low frequencies, $S_{\omega_{\mathrm{gbw}}}^{T(s)} \approx \frac{\alpha_{\mathrm{V}} s}{\omega_{\mathrm{gbw}}}$ and is very small. This means that variations of $\omega_{\mathrm{gbw}}$ have little influence on the overall gain. On the other hand, $S_{\alpha_{\mathrm{V}}}^{T(s)} \approx 1$ for low frequencies, which means that any variations of the feedback gain directly translates into variations of the overall gain. Thus the overall gain is set by the precision of the ratio of the feedback resistors, which can be very precise on chip. Note that the latter sensitivity can only get smaller for high frequencies, while the sensitivity to variations of the gainbandwidth product goes towards one for high frequencies. This means that the stabilising effect of feedback around a high-gain amplifier decreases with increasing frequencies. We can now find out at which frequency the contributions to the standard deviation

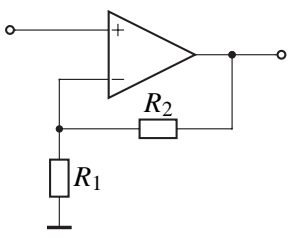

Figure 3: Low-gain voltage amplifier.

of $T(s)$ caused by the standard deviations of $\alpha_{\mathrm{V}}$ and of $\omega_{\mathrm{gbw}}$ become equal:

$$
\bar{\sigma}_{\alpha \mathrm{V}} S_{\alpha \mathrm{V}}^{T(s)}=\bar{\sigma}_{\omega_{\mathrm{gbw}}} S_{\omega_{\mathrm{gbw}}}^{T(s)} \Longrightarrow s=\omega_{\mathrm{gbw}} \frac{\bar{\sigma}_{\alpha_{\mathrm{V}}}}{\alpha_{\mathrm{V}} \bar{\sigma}_{\omega_{\mathrm{gbw}}}} .
$$

On CMOS ICs, resistor ratios can be precise to within $0.1 \%$, while the precision of $\omega_{\mathrm{gbw}}$ can be around $1 \%$. Thus, setting e.g. $\alpha_{\mathrm{V}}=2$, the variance of the $\omega_{\mathrm{gbw}}$ determines the variance of $\alpha_{\mathrm{V}}$ for frequencies above $\omega_{\mathrm{gbw}} / 20$, i.e., it normally dominates close to the pole frequency of a filter built with a feedback amplifier, and the matching precision of the feedback resistors is lost. Ideally, the gain variation is then $1 \%$ around the unity-gain frequency of the opamp and decreases with $20 \mathrm{~dB}$ per decade towards lower frequencies, but as with the resistances, some over-peaking occurs in practical cases. The feedback opamp can now be compared to CCIIs, whose current gains can also be precise to within $1 \%$. It then turns out that again, the feedback opamp is decidedly better than the CCII+ only for frequencies below $\omega_{\mathrm{gbw}} / 5$.

\section{WHY THE PERFORMANCE IS DIFFERENT}

Thus we can now explain why current-mode circuits are considered to be faster than voltage-mode circuits: although both would be similarly good from an ideal point of view, over-peaking caused by second-order effects makes problems close to the $\omega_{\mathrm{gbw}}$ product of the feedback opamps. It is important to see that the same overpeaking effects also occur in CCIIs that use local feedback to reduce the input resistance of the $\mathrm{X}$ terminal; in this case, the speed advantage of the CCII vanishes. It is also possible to build the circuit that is dual to the one in Fig. 3 using a current-mode opamp; its performance will then be similar. Furthermore, one can also build open-loop voltage amplifiers that show no over-peaking. However, it turns out that circuits without local stabilising feedback are just more typical for the current-mode approach.

Both the transfer function of the closed-loop opamp and the behaviour of the CCII are determined by the low-impedance nodes of the circuits only. These low-impedance nodes all look similar in both voltage-mode and current-mode circuits: a transistor $g_{\mathrm{m}}$ sets the node resistance, parasitic capacitances of transistors set the node capacitance, and the voltage swing is limited by transistors that would otherwise leave the region of saturation. Thus the non-dominant poles and zeros will be at similar frequencies, and the harmonic distortion at high frequencies and the noise properties will also be similar. What mainly determines the performance of a circuit is the number of low-impedance nodes and the way they are connected, i.e., the complexity of a circuit determines the performance of an amplifier or filter. As with feedback, one finds that less complex circuits are more typical for the current-mode approach, but there are a few voltage-mode circuits with reduced complexity, like the very fast $\mathrm{Gm}-\mathrm{C}$ filters presented in [10]. They 
were built with OTAs that only have input and output nodes, but no internal nodes at all. Of course, current-mode circuits can also be made more complex to improve their linearity and signal-to-noise ratio, but that slows them down again.

Thus the advantages of current-mode circuits that are often cited in the literature, like a potential for reaching higher frequencies, lower power consumption, and smaller chip area, are in fact real, but the reason is not technical, and has nothing to do with choosing voltages or currents to represent signals. The reasons for the difference are mainly the design preferences of the proponents of the current-mode approach (c.f. [3]).

\section{CONCLUSION}

While it is true that many current-mode circuits live up to the reputed advantages of the current mode, the reason is not that current has been used as a signal, but that circuit simplicity, lower power consumption and speed are often achieved at the cost of higher distortion, higher gain variation, and so on.

Therefore the work done in the many current-mode research groups also may give valuable insight to voltage-mode designers who need to build low-power, low-voltage, or high-speed circuits and are ready to trade in gain variations, distortion, or noise performance to achieve the goal. In the same way, the techniques used in classical voltage-mode design, like Miller feedback, offset compensation, and so on, can also be used in circuits that process current signals. We believe that the current-mode-voltage-mode divide that is so thoroughly described in [1] has now served its purpose, and conscious efforts should be made to re-integrate the current-mode community's knowledge into the main stream of IC design.

In spite of this conclusion, there will remain the question: What would happen if a designer set out to build a current-mode opamp that has approximately the same properties (CMRR, PSRR, linearity, chip area, etc.) as, e.g, the well-known opamp LM 741, and design it with the maximum possible speed? In the light of the above discussion, we believe that the speed would also be approximately the same, but until somebody tries this, which is not likely because the effort would be immense, the question will remain open.

\section{APPENDIX}

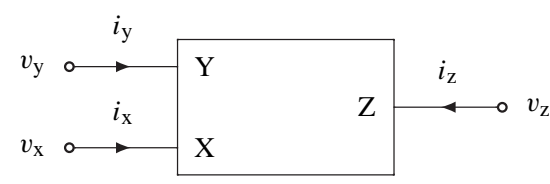

Figure 4: Symbol used for all types of current conveyors.

The second-generation current conveyor with current gain +1 , the CCII $+[11,12]$, is often drwan with the circuit symbol shown in Fig. 4. The CCII+ is described by three equations,

$$
i_{\mathrm{y}}=0, v_{\mathrm{x}}=v_{\mathrm{y}}, i_{\mathrm{z}}=i_{\mathrm{x}} .
$$

Various ways to implement current conveyors in CMOS and bipolar technologies are described in $[2,13]$.

\section{ACKNOWLEDGEMENTS}

I would like to thank Bram Nauta for a very interesting discussion on this subject.

\section{REFERENCES}

[1] Chris Toumazou, F. J. Lidgey, and D. G. Haigh, Analogue IC design: the current-mode approach, vol. 2 of IEE Circuits and Systems Series 2, Peter Peregrinus Ltd., 1990.

[2] Hanspeter Schmid, "Approximating the universal active element," IEEE Trans. CAS-II, vol. 47, no. 11, pp. 1160-1169, Nov. 2000.

[3] Hanspeter Schmid, "Theory and practice: Thinking styles in engineering and science," Australian Journal on Information Systems, pp. 106-115, Dec. 2001, Special issue on knowledge management. This paper is available from http://www.schmid-werren.ch/hanspeter/.

[4] Jirayuth Mahattanakul and Chris Toumazou, "Current-mode versus voltage-mode $G_{m}-\mathrm{C}$ biquad filters: What the theory says," IEEE Trans. CAS-II, vol. 45, no. 2, pp. 173-186, Feb. 1998.

[5] Alfonso Carlosena, Sonia Porta, and George S. Moschytz, "Current-mode realization of negative feedback SAB's," in 35th Midwest Symp. Circ. Sys., Washington, Aug. 1992, pp. 1296-1299.

[6] Gordon W. Roberts and Adel S. Sedra, "A general class of current amplifier-based biquadratic filter circuits," IEEE Trans. CAS-I, vol. 39, no. 4, pp. 257-263, Apr. 1992.

[7] Gordon W. Roberts and Adel S. Sedra, "All current-mode frequency selective circuits," Electronics Letters, vol. 25, no. 12, pp. 759-761, June 1989.

[8] B. Wilson, "Tutorial review: Trends in current conveyor and current-mode amplifier design," Int. J. Electronics, vol. 73, no. 3, pp. 573-583, 1992.

[9] Hanspeter Schmid and George S. Moschytz, "Fundamental frequency limitations in current-mode Sallen-Key filters," in Proc. ISCAS, Monterey, California, June 1998, vol. 1, pp. 57-60.

[10] Bram Nauta, "A CMOS transconductance-C filter technique for very high frequencies," IEEE J. Solid-State Circuits, vol. 27, no. 2, pp. 142-153, Feb. 1992.

[11] A. S. Sedra and K. C. Smith, "A second generation current conveyor and its applications," IEEE Trans. Circ. Theory, vol. 17, no. 1, pp. 132-134, Feb. 1970.

[12] A. S. Sedra, G. W. Roberts, and F. Gohh, "The current conveyor: history, progress and new results," IEE Proc., pt. G, vol. 137, no. 2, pp. 78-87, Apr. 1990.

[13] John Lidgey, Chris Toumazou, Alison Payne, Doug C. Wadsworth, Sittichai Pookaiyaudom, and Erik Bruun, "Tutorial 10: Current-mode analog signal processing; Part 2: current conveyors," in Proc. ISCAS, London, 1994, pp. 569641. 